(Aus dem Institut für exper. Pharmakologie der Universität Lemberg.)

\title{
Die Ungerinnbarkeit des Blutes bei der reflektorischen Tätigkeit der Speichel- drüsen und der Bauchspeicheldrüse. Das allgemeine Sekretionsgesetz der Verdauungssäfte ${ }^{1}$ ).
}

Von

Prof. Dr. L. Popielski,

Direktor des Instituts.

Eine Begleiterscheinung der Speichelsekretion bildet die Verminderung der Gerinnbarkeit des Blutes der Submaxillardrüse unter dem Einfluss der Reizung der Chorda tympani, des Nervus sympathicus unter der Einwirkung des Pilokarpins. Die durch den Einfluss von $\mathrm{BaCl}_{2}$ bewirkte Herabsetzung der Gerinnbarkeit des Blutes im ganzen Organismus verursacht die Sekretion aller Verdauungssäfte. Diese unter dem Einfluss von $\mathrm{BaCl}_{2}$ erfolgte Sekretion hat einen explosiven Charakter, und zwar infolge der Verengerung der Blutgefässe, welche die Drüsentätigkeit erschwert. Der Blutdruck bei Einwirkung von $\mathrm{BaCl}_{2}$ unterliegt Schwankungen, und diese spiegeln sich ebenfalls in der Sekretion der Säfte. Die durch Einführung von Vasodilatin $\left.{ }^{2}\right)^{3}$ ), Atropin ${ }^{4}$ ), Morphin ${ }^{4}$ ) und Urohämolysin ${ }^{5}$ ) in die Blutbabn bewirkte Ungerinnbarkeit des Blutes im ganzen Organismus wird gleichzeitig von einer grossen Dilatation der Blutgefässe der Bauchhöhle begleitet. Diese Erscheinungen gehen unmittelbar einer reichlichen Sekretion

1) Vorgelegt der Akademie der Wissenschaften in Krakau am 3. Juni 1912.

2) L. Po pielski, Über die physiologischen und chemischen Eigenschaften des Pepton Witte. Pflüger's Arch. Bd. 126 S.483. 1909.

3) L. Popielski, Über die physiologische Wirkung von Extrakten. Pflüger's Arch. Bd. 128 S. 191. 1909.

4) L. Popielski, Erscheinungen bei direkter Einführung von chemischen Körpern in die Blutbahn. Zentralbl. f. Physiol. Bd. 24 Nr. 24.

5) L. Popielski, Über die Eigenschaft des Harnes, den Blutdruck herabzusetzen. Zentralbl. f. Physiol. Bd. 24 Nr. 15. 
des Pankreas- und Magensaftes und der Galle voran. Dasselbe erhalten wir bei Bluttransfusion und beim anaphylaktischen Shock ${ }^{1}$ ). In diesen beiden Fällen begegnen wir einer reichlichen Sekretion des Pankreassaftes, gemäss einer stark ausgeprägten Ungerinnbarkeit des Blutes und einem stark herabgesetzten Blutdruck. Die Dilatation der Bauchgefässe allein verursacht keine Sekretion. Atropin bewirkt Erweiterung der Blutgefässe der Submaxillardrüse, ruft aber keine Speichelsekretion hervor, weil keine Verminderung der Blutgerinnbarkeit erfolgt. Ebenso führt die Reizung des $\mathrm{N}$. depressor die Erweiterung der Blutgefässe in der Bauchböhle herbei, ruft aber keine Pankreassekretion hervor, weil keine Blutungerinnbarkeit dabei vorhanden ist. Die Einführung des $\beta$-Imidazolyläthylamins in die Blutbahn erniedrigt ganz deutlich den Blutdruck, ruft aber keine Pankreassaftsekretion hervor, weil die Gerinnbarkeit des Blutes nicht nur nicht herabgesetzt, sondern sogar gesteigert ${ }^{2}$ ) wird.

In meiner letzten Arbeit $^{3}$ ) habe ich den Beweis geführt, dass die Sekretion des Pankreassaftes unter dem Einfluss von Vasodilatin auch dann zustande kommt, wenn der Blutdruck gleich Null ist. Die Sekretion wird in diesem Falle durch den in den Interzellularräumen der Bauchspeicheldrüse herrschenden Druck bewirkt. Diese Sekretion ist eben - wie ich es in der oben zitierten Arbeit nachzuweisen suche - eine "Filtration unter Druck".

In den von mir bis jetzt gewonnenen Tatsachen erfolgte die ekretion des Speichels oder des Pankreassaftes dort, wo neben der Erweiterung der Blutgefässe die Ungerinnbarkeit bzw. die Verminderung der Gerinnbarkeit des Blutes auftrat. Unerforscht blieben aber die Bedingungen der unter dem Einfluss mancher als Sekretionsmittel bekannten Körper und reflektorisch auftretenden Tätigkeit der Speicheldrüsen und des Pankreas.

In der vorliegenden Arbeit befasse ich mich mit der Bestimmung der Blutgerinnbarkeit bei der Tätigkeit der Speichel- und der Pankreasdrüse auf reflektorischem Wege und bei der Tätigkeit der Speicheldrüsen unter dem Einfluss des Muskarins.

1) G. Modrakowski, Über die Grunderscheinungen des anaphylaktischen Shocks. Arch. f. exper. Path. u. Pharm. Bd. 69 S. 67. 1912.

2) G. Modrakowski, 1. c. S. 75 .

3) L. Popielski; Blutdruck und Ungerinnbarkeit des Blutes bei der Tätigkeit der Verdauungsdrüsen. Bull. de l'Acad. des Sciences de Cracovie, sciences naturelles, Série B 1911 p. 727 und Pflüger's Arch. Bd. 144 S. 135.1912. 


\section{Die Blutgerimnbarkeit bei der reflektorischen Tätigkeit der Speicheldrüsen und des Pankreas.}

Die Bestimmung der Blutgerinnbarkeit führte ich nach der von mir in der oben zitierten Arbeit beschriebenen Methode von $\mathrm{Br}$ odie aus. Um eventuelle methodische Fehler zu verhüten und die erhaltenen Ergebnisse als sichere Tatsachen hinstellen zu können, wurde die Bestimmung der Blutgerinnbarkeit in vielen Fällen zugleich von mir und meinem Assistenten Dr. Czubalski') vorgenommen.

Die vorher ausgeführten Kontrollversuche zeigten bei unseren Bestimmungen eine Differenz von höchstens $20 "-30$ ". Ausserdem fing ich bei der Untersuchung des Blutes aus der Pankreasdrüse dasselbe gleichzeitig in eine Eprouvette auf und führte in derselben in üblicher Weise die Bestimmungen der Gerinnbarkeit aus.

Da die Differenzen in der Gerinnbarkeit des Blutes der ruhenden und der tätigen Drüse stark ausgeprägt waren, liess sich mittels der früher angewandten, überhaupt ungenauen Methode nur eine Verminderung der Gerinnbarkeit feststellen, und erst mit Hilfe der Methode von Brodie konnte genaue Bestimmung vorgenommen werden.

\section{Die Speicheldrüsen.}

Die Untersuchungen führte ich an der Submaxillardrüse aus. In den Whartonian'schen Gang wurde eine Kanüle zwecks Bestimmung der Speichelsekretion und in die V. submaxillaris externa eine Kanüle zum Auffangen des Blutes eingeführt. Um die Speichelsekretion anzuregen, wurde in die Mundhöhle des Hundes $0,4 \%$ ige $\mathrm{HCl}$ gegossen. Den Verlauf der Blutgerinnbarkeit während der reflektorischen Tätigkeit der Drüse zeigt folgender Versuch.

23. Januar 1912. Hund von $11 \mathrm{~kg}$ Gewicht. Das Tier wurde durch Einführung von. $3 \mathrm{ccm}$ einer $1 \%$ igen Kurarelösung in die Blutbahn immobilisiert. Diese Methode ist nicht zweckentsprechend, weil, wie $\mathrm{Czubals} \mathrm{ki}^{1}$ ) nachgewiesen hat, das Blut ungerinnbar und der Blutdruck herabgesetzt wird. Will man den Versuch ausführen, muss man ziemlich lange warten, bis die Gerinnbarkeit des Blutes wiederum zur Norm zurückgekehrt ist. Die Durchtrennung des Rückenmarks kann nicht angewendet werden, weil der Blutdruck bedentend sinkt und

1) F. Czubalski, Über den Einfluss von Curare auf die Verdauungsdrüsen und die Gerinnungsfähigkeit des Blutes. Pflüger's Arch. Bd 183 S. 225. 1910 . 
das Blut infolgedessen nur sehr langsam aus der Kanüle herausfliesst, so dass es unmöglich ist, Blut behufs Ausführung der Bestimmungen aufzufangen.

Die Bestimmung der Gerinnbarkeit des Blutes nach Einführung von Curare wurde von Zeit zu Zeit ausgeführt. Die erhaltenen Zahlen sind folgende:
1. $14^{\prime} 14^{\prime \prime}$,
2. $9^{\prime} 00^{\prime \prime}$,
3. $8^{\prime} 03^{\prime \prime}$,
4. $5^{\prime} 55^{\prime \prime}$.

Erst zu dieser Zeit wurde die 0,4\% ige HCl-Lösung in die Mundhöhle des Versuchstieres gegossen. Das während der 100 Millimeterteilstriche in $1^{\prime}$ betragenden Speichelsekretion zur Bestimmung aufgefangene Blut gerinnt nach $10^{\prime} 25^{\prime \prime}$, bei 60 Teilstrichen in $1^{\prime}$ nach $8^{\prime} 00^{\prime \prime}$; bei noch geringerer Sekretion gerinnt es nach $7^{\prime} 40^{\prime \prime}$ und $7^{\prime} 18^{\prime \prime}$. Nachdem die Sekretion ganz aufgehört hat, gerinnt das Blut nach $5^{\prime} 25^{\prime \prime}$.

Wie aus dem eben angeführten Versuche zu ersehen ist, sind die Unterschiede der Gerinnbarkeit des Blutes in der Norm und während der reflektorischen T'ätigkeit der Drüse ganz ausgesprochen. In der Norm gerinnt das Blut im obigen Versuche nach $5^{\prime} 25^{\prime \prime}$, während der Tätigkeit der Drüse nach $10^{\prime} 25^{\prime \prime}$. Es muss hier bemerkt werden, dass gleichzeitig mit diesem Phänomen während der reflektorischen Tätigkeit der Drüse auch gesteigerte Schnelligkeit und eine deutliche Veränderung der Eigenschaften des Blutes auftreten. Das Blut fliesst aus der Vene nicht nur schneller heraus, sondern es wird stark rot, arteriell. Man wird wohl begreifen, dass infolge der Erweiterung der Blutgefässe nur in der Drüse der Blutdruck in den Kapillargefässen steigt und hierdurch der Druck, unter welchem die Sekretion erfolgt, ebenfalls gesteigert wird.

\section{Die Bauchspeicheldrüse.}

Das Phänomen der verminderten Blutgerinnbarkeit tritt bei der reflektorischen Tätigkeit der Bauchspeicheldrüse noch deutlicher hervor.

Die Versuche wurden an Hunden, denen das Rückenmark durchgeschnitten worden war, ausgeführt. Das Blut zur Untersuchung wurde der Pankreasvene an der Stelle entnommen, wo die Bauchspeicheldrüse sich vom Duodenum entfernt. In zwei Versuchen wurde das Blut durch Venenpunktion gewonnen. In einem Versuche führte ich Kanülen in die Vene in ähnlicher Weise ein, wie ich es bei der Untersuchung der Speicheldrüsen immer tat; in einem anderen Versuche endlich kam in die Vene eine Glaskanüle, durch welche ich zuvor die Jugularvene desselben Hundes durchgezogen und nach Umstülpung an beiden Enden abgebunden hatte. Diese Versuchsanordnung wurde deshalb getroffen, damit das aus der Vene heraus- 
fliessende Blut mit der normalen Gefässwand in Berührung komme und hierdurch ein mehrmaliger Wechsel der Kanüle vermieden werde.

Die von mir erhaltenen Resultate sind aus folgenden Versuchen zu ersehen:

25. Jannar 1912. Hund von $11 \mathrm{~kg}$ Gewicht. Durchschneidung des Rückenmarks. Künstliche Atmung. Das der Pankreasvene durch Punktion entnommene Blut gerinnt in der Norm nach $7{ }^{\prime} 18^{\prime \prime}$.

Zeit Das Niveau

in der Röhre

Um 12 h $57^{\prime}$

12 h $58^{\prime} \quad 58$

12 h $59^{\prime} \quad 59$

$1 \mathrm{~h} 00^{\prime} \circ 59$

$1^{\text {h }} 02^{\prime} 10^{\prime \prime} \quad 60$ Einführung in das Duodenum von $40 \mathrm{ccm}$ $0,36 \%$ igem $\mathrm{HCl}$.

1 h $03^{\prime} \quad 65$

1 h $04^{\prime} \quad 84$

1 h $05^{\prime} \quad 135$

$1 \mathrm{~h} 06^{\prime} \quad 225$

1 h $06^{\prime} 30^{\prime \prime} \quad 275$

1 h $07^{\prime} \quad 0 \quad$ Kanüle wird gewechselt.

1 h $08^{\prime} \quad 102$

1 h $09^{\prime} \quad 224$

$1^{\text {h }} 10^{\prime} 30^{\prime \prime} \quad 0$ Das Blut wird entnommen; es gerinnt nach

1 h $11^{\prime} \quad 125$

1 h $12^{\prime} 30^{\prime \prime} \quad 15$ (Kanüle wird gewechselt.)

1 b $13^{\prime} \quad 70$

1 h $13^{\prime} 30^{\prime \prime} \quad 120$ Die Sekretion vermindert sich allmählich und

um $1^{\text {h }} 38^{\prime} \quad 0$

1 h $39^{\prime} \quad 0$

1 h $40^{\prime} \quad 1$

1 h $48^{\prime} \quad 10$ Das zur Untersuchung entnommene Blut gerinnt nach $7^{\prime} 25^{\prime}$.

2. April 1912. Hund von $18 \mathrm{~kg}$ Gewicht. Durchschneidung des Rückenmarks. Künstliche Atmung. Durchschneidung der Nervi vagi. Pankreasfistel. In $1^{\prime}$ werden ungefähr zwei Teilstriche sezerniert.

Ohne das Versiegen der Sekretion abzuwarten, wurde das Blut zur Untersuchung genommen. Das Blut gerinnt nach $13^{\prime} 41^{\prime \prime}$.

Um 12 h $30^{\prime}$ wurden in das Duodenum $40 \mathrm{~cm} 0,36 \%$ igem $\mathrm{HCl}$ eingefübrt.

Zeit Das Niveau des Pankreas-

Um $12^{\text {h }} 33^{\prime} \quad 20$

$12^{\mathrm{h}} 34^{\prime} \quad 40$ 
L. Popielski:

Zeit Das Niveau des Pankreas-

2 म $35^{\prime} \quad 62$

12 h $36^{\prime} \quad 70$

12 h $37^{\prime} \quad 85$

$12^{\mathrm{h} 38^{\circ}} \quad 100$ Das Blut wurde entnommen; es gerinnt nach $17^{\prime} 42^{\prime \prime}$ (Czubalski), nach $18^{\prime} 00^{\prime \prime}$ (Popielski).

Um $1^{\text {h }} 18^{\prime}$ hört die Sekretion gänzlich auf.

1 h $18^{\prime} \quad 62$

1 h $19^{\prime} \quad 63$

1 h $20^{\prime} \quad 63$

1 h $22^{\prime} \quad 63$

1 h $26^{\prime} \quad 68$ Das Blut wurde entnommen; "es gerinnt nach

$8^{\prime} 40^{\prime \prime}$ (Popielski), nach 9'10"(Czabalski).

Um $1 \mathrm{~h} 55^{\prime}$ wurden $10 \mathrm{ccm} 1 \%$ iges Atropin. sulfur. subkutan eingespritzt.

Um $2 \mathrm{~h}^{\circ} 0^{\prime} \quad 0 \quad$ Die Pupillen sind weit; in das Duodenum warden $40 \mathrm{ccm} \quad 0,36 \%$ iges $\mathrm{HCl}$ eingeführt.

$2^{\text {h }} 22^{\prime} 30^{\prime \prime} \quad 0$

2 h $23^{\prime} \quad 15$

2 h $24^{\prime} \quad 50$

2 h $25^{\prime} 30^{\prime \prime} \quad 72$

2 h $26^{\prime} \quad 120$

2 h $27^{\prime} \quad 152$

$2^{\text {h }} 27^{\prime} 30^{\prime \prime} \quad 192$

$3^{\mathrm{h}} 15^{\prime} \quad 110$

3 h $20^{\prime} \quad 115$

3 h $23^{\prime} \quad 116$

3 h $40^{\prime} \quad 140$

4 h $37^{\prime} \quad 168$

$4^{\text {h }} 57^{\prime}$

5 h $20^{\prime}$

5 h $35^{\prime}$

Um $\quad 5$ h $55^{\prime}$

5 h $56^{\prime}$

5 h $57^{\prime}$

5 h $58^{\prime}$

5 h $59^{\prime}$

6 h $^{\circ} 00^{\prime}$

$6{\mathrm{~h} 01^{\prime}}^{\prime}$

186

40

105 .

176

246

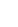

50

(1)

2 Blut wurde entnommen; es gerinnt nach $17^{\prime} 20^{\prime \prime}$.

Die Selkretion vermindert sich allmählich.

Blut wird entnommen; es gerinnt nach $13^{\prime} 05^{\prime \prime}$.

173 Blut wird entnommen; es gerinnt nach $13^{\prime}$ (Czubalski), 13' 13" (Popielski).

188 Aus der Vene werden in einen Glaszylinder innerhalb 15" 4,5 ccm Blut abgelassen. Das Blut gerinnt in der Eprouvette nach $8^{\prime}$.

0 In das Duodenum werden $40 \mathrm{ccm} 0,4 \%$ ges $\mathrm{HCl}$ eingeführt.

0 (Die Kanüle wird gewechselt.)

72 In Eprouvetten aufgefangenes Blut gerinnt nach $20^{\prime}$. 
Die Ungerinnbarkeit des Blutés bei der reflektor. Tätigkeit etc.

\begin{tabular}{|c|c|c|}
\hline $\begin{array}{r}\text { Das } \\
\text { sa }\end{array}$ & $\begin{array}{l}\text { eau des } \\
\text { in der }\end{array}$ & $\begin{array}{l}\text { Pankreas- } \\
\text { tlasröhre }\end{array}$ \\
\hline 6 h $02^{\prime}$ & 142 & \\
\hline 6 h $03^{\prime}$ & 212 & \\
\hline $6^{\text {h }} 04^{\prime}$ & 55 & (Die Kanüle wird gewechselt.) \\
\hline 6 b $05^{\prime}$ & 121 & \\
\hline 6 h $05^{\prime} 30^{\prime \prime}$ & 236 & \\
\hline
\end{tabular}

Aus den angeführten Versuchen ist leicht $\mathrm{zu}$ ersehen, dass während der reflektorischen Tätigkeit der Drüse die Gerinnbarkeit des Blutes sich bedeutend vermindert. Nachdem die Sekretion fast gänzlich aufgehört hatte (ein Teilstrich - in $5-6$ Minuten), gerann das Blut nach $8^{\prime} 40^{\prime \prime}$. Während der Sekretion, welche zwei Teilstriche in der Minute beträgt, gerinnt das Blut nach 13' 41", $13^{\prime} 05^{\prime \prime}$, $13^{\prime} 00^{\prime \prime}$ in verschiedenen Stadien des Versuches (am Anfange und am Ende). Während der Sekretion, welche 15-20 Teilstriche in einer Minute beträgt, erfolgt die Gerinnung des Blutes nach $17^{\prime} 42^{\prime \prime}$, $17^{\prime} 20^{\prime \prime}$ und während der 70 Teilstriche in einer Minute betragenden Sekretion nach 20'. Die letzte Bestimmung darf nicht mit den vorhergehenden verglichen werden, weil sie nicht nach der Methode von Brodie, sondern auf übliche Weise direkt in der Eprouvette ausgeführt wurde. Das Atropin übt keinen Einfluss auf die Sekretion des Pankreassaftes oder auf die Gerinnbarkeit des Blutes aus.

Diese Tatsachen weisen darauf hin, dass während der sekretorischen Funktion der Drüse regelmässig zwei Phänomene, d. h. die Erweiterung der Blutgefässe und die Verminderung der Blutgerinnbarkeit auftreten.

Ich wandte mich hierauf zu den Körpern, welche ähnlich wie Pilokarpin eine Speichelsekretion hervorrufen. Ich verwendete vorerst Muskarin, welches die sekretorische Funktion der Speicheldrüsen entschieden steigert. Die Ergebnisse meiner Untersuchungen sind im folgenden Experimente veranschaulicht:

20. Mai 1912. Hund von $11 \mathrm{~kg}$ Gewicht. In Chloroformnarkose wurde in den Whartonian'schen Gang eine Kanüle eingeführt und auch eine kleine Kanüle in die Vena maxillaris externa (zum Auffangen des Blutes) hineingeschoben; die Chorda tympani wurde durchgeschnitten. Die Speichelausscheidung war sehr gering, sie betrug ungefähr 1 Teilstrich in $4-5$ Minuten. Die Blutgerinnbarkeit war in vier Bestimmungen wie folgt: $1.8^{\prime} 15^{\prime \prime}, 2.8^{\prime} 30^{\prime \prime}$, 3. $7^{\prime} 32^{\prime \prime}, 4.8^{\prime} 00^{\prime \prime}$.

Um $7 \mathrm{~h} 04^{\prime}$ wurden subkutan $6 \mathrm{ccm}$ einer $0,1 \%$ igen Muskarinlösung eingeführt. (Synthetisches Muskarin mit schwachem Methylamingeruch, befindet sich seit einigen Jahren im Laboratorium.) 


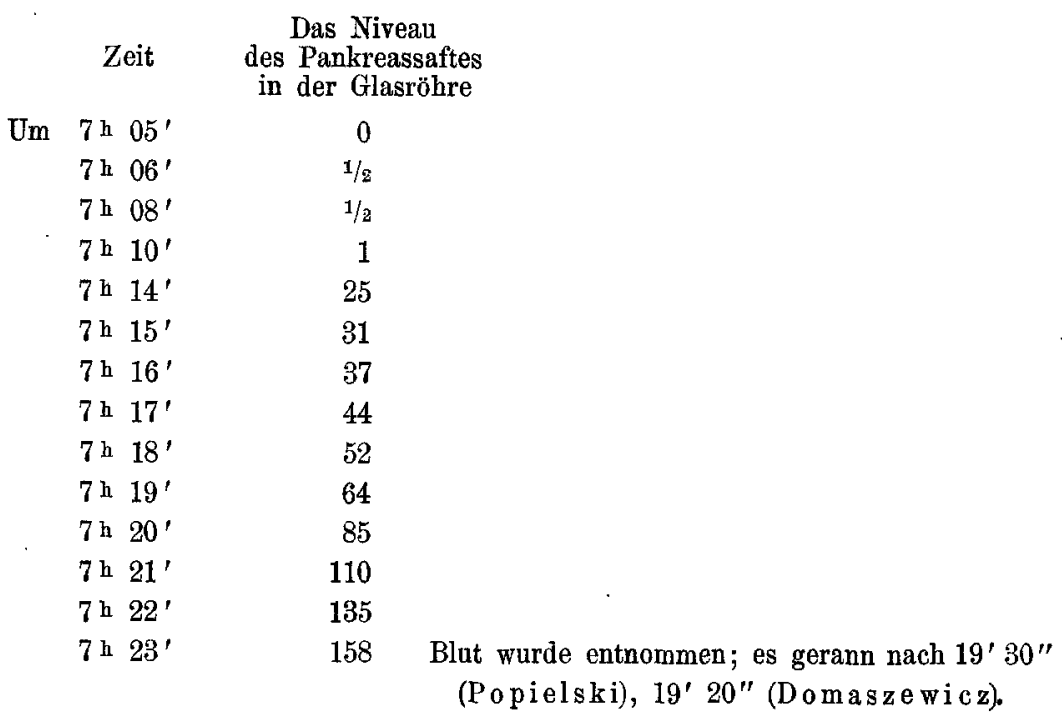

Wie man aus den obigen Versuchen ersieht, ist der Untersehied im Blutgerinnen ausserordentlich gross. Im inaktiven Zustande der Drüse gerinnt das Blut nach acht Minuten, im aktiven durch das Muskarin hervorgerufen dagegen erfolgt die Gerinnung erst nach 19 Minuten. Die Einführung des Atropins hebt sowohl die Erscheinung der Sekretion als auch die Erscheinung der verminderten Gerinnbarkeit auf. Nach Einführung des Atropins gerinnt das Blut nach $6^{\prime} 35^{\prime \prime}$.

In dem Versuche mit Muskarin verdienen folgende Tatsachen Beachtung: das aus der Drüsenvene herausfliessende hellrote Blut und das bedeutend schnellere Herausfliessen desselben aus der Vene. Die Schnelligkeit des Blutausfliessens habe ich nicht genauer bestimmt, es war jedoch diese Erscheinung sehr auffallend.

Während die Vertiefung in der Hautwunde im normalen $\mathrm{Zu}$ stande sich während 50" mit Blut ausfüllte, geschah dies bei Anwendung von Muskarin während $6-8$ ".

Während der Speichelsekretion, welche in einer Minute 15 Teilstriche betrug, gerann das Blut nach $13^{\prime} 6^{\prime \prime}$. Als aber die Speichelausscheidung vollständig aufgehört hatte, d. h. als das Atropin zu wirken begann, erfolgte Blutgerinnung nach $6^{\prime} 35^{\prime \prime}$. Gleichzeitig war das Blut bedeutend dunkler.

Unter der Wirkung von Nikotin ist die Speichelausscheidung von sehr kurzer Dauer und die Blutentnahme deshalb gerade während 
der Ausscheidung sehr erschwert. Wiederholte Nikotineinführung ruft keine Ausscheidung mehr hervor. Nach der kurzen Ausscheidungsperiode unter der Wirkung des Nikotins hört die Sekretion vollständig auf. In einem Versuche gerann das Blut vor Einführung des Nikotins bei geringer Speichelsekretion nach zehn Minuten; nach Einführung des Nikotins während der Periode des vollständigen Ausbleibens der Sekretion gerann das Blut nach 6 '.

In den von mir beschriebenen Versuchen tritt die Sekretion des Speichels und des Pankreassaftes immer bei Vorhandensein von zwei Bedingungen auf: der verminderten Blutgerinnbarkeit und Erweiterung der Blutgefässe. Die Bedeutung dieser beiden Bedingungen ist ganz verständlich. Die flüssigen Blutteile können in die Interzellularräume der Drüse nur durch die erweiterten Gefässe gelangen.

Die Bedeutung der Abnahme der Blutgerinnbarkeit wird vollkommen verständlich bei Heranziehung des Vergleiches mit Stärkekleister. Ein Filter, auf dem sich Stärkekleister befindet, besitzt unvergleichlich grössere Poren als die Wände der Blutgefässe. Selbst bei ziemlicher Dünnflüssigkeit geht jedoch der Kleister durch die Filterporen nicht hindureh; das erfolgt erst, wenn er sich noch weiter verflüssigt, wie es bei der Verdaung des Kleisters durch ein amylolytisches Ferment, z. B. Ptyalin, geschieht.

Wenn das Blutgefäss z. B. durch Einführung von Adrenalin verengert wird, findet eine Säfteausscheidung nicht statt. Die Reizung der Nervi sympathici ruft eine Speichelausscheidung dann hervor, wenn die Verengerung der Blutgefässe einèr Erweiterung derselben Platz macht.

Damit die flüssigen Blutteile durch die Blutgefässwände hindurchtreten, ist eine verringerte Blutgerinnbarkeit erforderlich. Normal gerinnendes Blut würde beim Durchtritt durch die mikroskopisch engen Gefässwandräume sofort ein Fibrinnetz bilden. Daher findet auch unter normalen Bedingungen kein Durchsickern der flüssigen Blutteile in die Interzellularräume der Drüse statt, und diese schwillt auch nicht an. Das Durchsickern der flüssigen Blutteile ist aber möglich, und erklärlich ist diese Erscheinung nur durch Verringerung der Blutgerinnbarkeit. Kleine Schwankungen in der Blutgerinnbarkeit können bereits die Ausscheidung bewirken.

Bei Einführung von Vasodilatin erfolgt die Sekretion des Pankreassaftes sogar dann, wenn die Blutgerinnbarkeit nur um 2 Minuten gegenüber der Norm verlängert ist. Die Grösse der 
Ausscheidung läuft parallel mit dem Grade der Ungerinnbarkeit des Blutes. Je stärker die Ungerinnbarkeit hervortritt, desto grösser ist die Ausscheidung des Pankreassaftes.

Die Absonderung der Verdauungssäfte lässt sich, wie ich gezeigt habe, auf Filtration unter Druck zurückführen. Bei dieser Filtration sind jedoch auch die Drüsenzellen, durch welche der flüssige Anteil des Blutes passiert, beteiligt. Je langsamer die Filtration erfolgt, desto grösser wird der Einfluss der Drüsenzellen auf die Zusammensetzung des Saftes sein. Es lassen sich nun Bedingungen schaffen, unter denen die Filtration so schnell erfolgt, dass das Sekret ausschliesslich mineralische Bestandteile enthält. Das fand in den Versuchen von Dr. Mazurkiewicz ${ }^{1}$ ) statt. Bei einem sehr starken auf die Schleimhaut des Duodenums wirkenden Reize (es war $0,5 \% \mathrm{HCl}$ in den Magen eingeführt) wurde ein Pankreassekret mit einem Gehalte von $0,92 \%$ festen Bestandteilen, davon $0,9 \%$ mineralische, erhalten. Mit anderen Worten, der Saft war fast frei von organischen Bestandteilen, also ein wirkliches Filtrat des Blutes. Wir haben es daher hier mit einer Erscheinung von rein physikalischer Natur zu tun. Es ist nicht leicht, bei physiologischen Versuchen Bedingungen zu schaffen, unter denen nur physikalische Kräfte wirksam sind. Solche Bedingungen gelang es mir bei den Versuchen mit Komprimierung der Brustaorta nach vorheriger Injektion von Vasodilatin in Gestalt von Pepton Witte zu schaffen Hierbei erfolgte die Pankreassaftabsonderung bei einem Blutdruck $=0$, wobei das Sekret nach meinen letzten Bestimmungen bis zur Höhe von $90 \mathrm{~mm}$ anstieg, was einem Druck von $7 \mathrm{~mm} \mathrm{Hg}$ über 0 entspricht.

Die den Pankreassaft zu dieser Höhe antreibende Kraft konnte nicht von selbst entstehen, sondern musste ihren Ursprung in der Blutdrucksenkung haben.

Leicht zu beantworten ist die Frage, bei welchem absoluten Blutgerinnbarkeitsgrade (nach der Brod i e'schen Methode) die Ausscheidung erfolgt.

Offenbar hat die Weite der Blutgefässe einen Einfluss auf den Ausscheidungseffekt. Auf Grund meiner eigenen Untersuchungen

1) W. Mazurkiewicz, Die festen Bestandteile des'Bauchspeichels und die Theorie der Sekretionstätigkeit des Pankreas. Pflüger's Arch. Bd. 121 S. 75. 1908. 
kann ich nur feststellen, dass beim Blutgerinnen wach $10^{\prime}$ eine ganz deutliche Speichelsekretion auch dann stattfindet, wenn die Chorda tympani und die Nervi sympathici durchschnitten sind. Die Ausscheidung ist gering, da auf diese Erscheinung auch die Weite der Blutgefässe einen Einfluss ausübt.

Dasselbe gilt für die Pankreasdrüse. Wenn wir eine grosse Dosis von Vasodilatin einführen, um eine bedeutende Verminderung des Blutdrucks herbeizuführen, so dauert eine energische Sekretion höchstens 14'. Eine geringe Ausseheidung dauert dagegen so lange, als die Blutgerinnbarkeit verringert und die Blutgefässe erweitert sind, und sie hört auf, sobald der Blutdruck hochgradig gesteigert wird.

Während des Erstickens findet Ausscheidung des Speichels, doch nicht die von Pankreassaft statt, und zwar deshalb, weil die Pankreasblutgefässe verengert sind. Der Blutdruck steigt während des Erstickens infolge der Verengerung der Blutgefässe, hauptsächlich derjenigen der Bauchhöhle. Wenn man jedoch, wie ich es in meinen Versuchen getan habe, das Rückenmark unterhalb der Medulla oblongata durchschneidet, um auf diese Weise die Möglichkeit der Verengerung der Blutgefässe der Bauchhöhle aufzuheben, dann erfolgt beim Ersticken auch deutlich die Sekretion des Pankreassaftes.

Wenden wir uns nun der Frage nach der Gerinnbarkeitsgrenze zu, bei der keine Ausscheidung stattfindet. Aus meinen Untersuchungen ergibt sich nun auch, dass bei Blutgerinnbarkeit von ungefähr $6-7^{\prime}$ nach Brodie keine Sekretion stattfindet. In den von mir ausgeführten Untersuchungen ist die Verringerung der Blutgerinnbarkeit eine der unentbehrlichsten Bedingungen für die Sekretionstätigkeit der Drüsen. Diese verringerte Blutgerinnbarkeit beobachten wir ausserhalb des Organismus unter Bedingungen, die für das Blut nicht normal sind, während der die Ungerinnbarkeit des Blutes bewirkende Körper zerfällt. Daher kommt es, dass das Blut nach einiger Zeit gerinnt. Andererseits benützen wir bei unseren Versuchen immer das durch die Drüse hindurchfliessende Blut, welches den seine Ungerinnbarkeit bewirkenden Körper in sich trägt. Wir haben es daher mit einer Mischung des normalen, immer durch die Drüse hindurchfliessenden und ees die Eigenschaft der Ungerinnbarkeit annehmenden Blutes zu tun. Es ist daher klar, dass solches Blut nach einiger Zeit gerinnen muss. Mit anderen Worten, das normale Blut beschleunigt das Gerinnen des Blutes, weil jener 
Körper, welcher die Ungerinnbarkeit bewirkt, zerfällt. Wir besitzen hierfür Beweise, wenn auch nur indirekte.

Wenn man während des anaphylaktischen Shocks dem Hunde im Augeublick des niedrigsten Druckes Blut entnimmt, so gerinnt es während 2-3 Tagen nicht. Wenn wir nun das Plasma dieses nicht gerinnenden Blutes zu normalem Hundeblute zusetzen, wird das letztere viel langsamer gerinnen. Wenn wir z. B. 1/4 ccm Serum zu $5 \mathrm{ccm}$ des normalen Blutes hinzufügen, gerinnt dieses Blut nach $39^{\prime}$.

Das Blut gerinnt:

bei Hinzugabe von ${ }^{1 / 2} \mathrm{ecm}$ Serum zu 5 cem normalem Blut nach 1 h $20^{\prime}$,

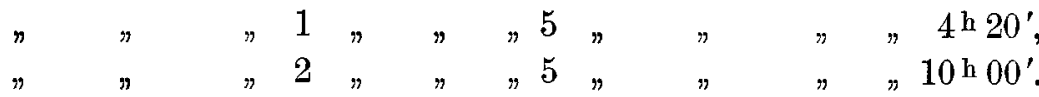

Reines Blut gerinnt nach 8 Minuten.

Es ist also aus diesem Versuche zu ersehen, dass im normalen Blut der Körper, welcher die Ungerinnbarkeit bewirkt, zerfällt, und dass infolgedessen das Blut gerinnt. Es zerfällt auch jener Körper, welcher in unseren Versuchen das Gerinnen des Blutes verlangsamt. Beide Körper entstehen höchstwahrscheinlich im Endothelium der Blutgefässe während des anaphylaktischen Shocks, in den Gefässen der Bauchhöhle während der Sekretion, in den mitgeteilten Versuchen im Endothelium der Blutgefässe der arbeitenden Drüse. Es lässt sich selbstverständlich daraus noch kein Schluss auf die Identität der betreffenden Körper ziehen, man darf sie nur für sehr wahrscheinlich halten, und zwar um so mehr, da beide Körper die gleichen Sekretionserscheinungen herbeiführen. Wir werden also verstehen, weshalb in unseren Experimenten das Blut nach einiger Zeit gerinnt.

Die Ausscheidung des Pankreassaftes bei Einführung von Morphin ${ }^{1}$ ), Atropin ${ }^{1}$ ), Vasodilatin $^{2}$ ), Urohämolysin ${ }^{4}$ ), Curare $^{3}$ ) in die Blutbahn und bei Transfusion ${ }^{5}$ ) des Blutes ist identisch mit

1) l. c.

2) $1 . \mathrm{c}$.

3) 1. c.

4) $1 . \mathrm{c}$.

5) J. Studziński, Über die giftigen Eigenschaften des Blutes. Zentralbl. f. Physiol. Bd. 23 H. 22. 
der Sekretion während des anaphylaktischen Shocks ${ }^{1}$ ). Man kann daher behaupten, dass der die Ungerinnbarkeit hervorrufende Körper in allen diesen Fällen derselbe ist. Jedenfalls ist für uns gegenwärtig die Tatsache von Bedeutung, dass die Tätigkeit der Verdauungsdrüsen (der Speicheldrüsen, der Bauchspeicheldrüse) als Ausdruck der geringeren Blutgerinnbarkeit und Erweiterung der Blutgefässe auftritt. Auf welche Ursachen auch diese Erscheinungen zurückgeführt werden mögen, immer begegnen wir der Sekretion, als einer notwendigen, unvermeidlichen Erscheinung.

Meine Untersuchungen führen mich zu dem Schluss, dass der die Ungerinnbarkeit des Blutes bewirkende Körper sich während der Drüsentätigkeit im Endothelium der Kapillargefässe der Drüsen bildet. Es ist vollständig klar, dass in den Endothelzellen selbst Veränderungen auftreten können, infolge deren der erwähnte Körper entstehen und eine Sekretion hervorrufen kann.

Im Lichte meiner Untersuchungen wird die paralytische Speichelausscheidung nach Durchschneidung der Chorda tympani verständlich. Die Ausscheidung erfolgt nicht sogleich nach der Durchschneidung, sondern erst nach einer Zeit, so dass wir erst nach etlichen Tagen eine mehr oder weniger ergiebige Sekretion beobachten können. Höchst wahrscheinlich haben wir es hier mit Degenerationsprozessen zu tun, welche, längs des Nerven fortschreitend, auch auf die Endothelzellen übergreifen. Infolge der Degenerationsprozesse bildet sich in den Zellen der die Ungerinnbarkeit des Blutes hervorrufende Körper, der die Ausscheidung herbeiführt.

Nach Anlegen einer Pankreasfistel tritt bei manchen Hunden nach Verlauf von 2-3 Wochen eine reichliche Saftausscheidung auf. Man darf vermuten, dass infolge postoperativer chronischer Entzündungsprozesse bei gewissen Hunden Veränderungen im Endothel der Gefässe auftreten, und dass dieselben die Bildung des die Ungerinnbarkeit des Blutes bewirkenden Körpers verursachen.

In manchen Fällen der Hypersekretion der Magendrüsen darf man vermuten, dass im Endothel der Kapillargefässe Veränderungen eingetreten sind, welche den oben erwähnten Veränderungen in der Pankreasdrüse ähnlich sind. Die von mir angeführten Erscheinungen der Hypersekretion der Drussen verlieren ihre Rätselhaftigkeit, werden klar und verständlich und weiteren Untersuchungen zugänglich.

1) G. Modrakowski, l. c. S. 71 und 72 . 
14 L. Popielski: Die Ungerinnbarkeit des Blutes bei der refl.Tätigkeit etc.

In meinen Untersuchungen befasste ich mich mit Versuchen an zwei am meisten zugänglichen Drüsen: mit der Submaxillar- und der Bauchspeicheldrüse. Man darf jedoch voraussetzen, dass die von mir beobachteten Erscheinungen allen Verdauungsdrüsen und vielleicht allen Drüsen überhaupt gemeinsam sind.

Die Magendrüsen sind unter den gleichen Bedingungen tätig wie die Bauchspeicheldrüse. Die Einführung von Morphin und Vasodilatin in die Blutbahn ruft ebenfalls eine Ausscheidung des Magensaftes hervor. Dieselben Nerven verursachen bei ihrer Reizung die Sekretionstätigkeit des Magens. Eine Verallgemeinerung erscheint also gerechtfertigt.

So gelangen wir denn zu dem der Ausscheidungstätigkeit der Verdauungsdrüsen zugrunde liegenden allgemeinen Gesetze: Die Sekretionstätigkeit ist eine Folge der Ungerinnbarkeit des Blutes und der Erweiterung der Blutgefässe. 